\title{
The Use of Thermal Imaging in Solving Problems of Modeling Radar Images
}

\author{
Igor N. Ischuk ${ }^{\mathrm{a}, \mathrm{b}}$, Valery N. Tyapkin ${ }^{\mathrm{b}}$, \\ Konstantin V. Postnov*a and Sergey A. Panov ${ }^{\text {a }}$ \\ ${ }^{a}$ Military Education and Research Centre of Military-Air Forces \\ «Military-Air Academy \\ Named After Professor N.E. Zhukovsky and Yu.A. Gagarin» \\ 54a Starykh Bolshevikov Str., Voronezh, 394064, Russia \\ ${ }^{b}$ Siberian Federal University \\ 79 Svobodny, Krasnoyarsk, 660041, Russia
}

Received 16.01.2017, received in revised form 09.02.2017, accepted 27.02.2017

The article presents a technique to obtain radar images of the Earth surface by converting thermal tomography image based on the functional dependence of the dielectric constant materials on their thermal properties. Representation of bond absorption coefficient of electromagnetic waves and thermal insulator characteristics.

Keywords: thermal tomography, model radar image, the reflection coefficient, the dielectric constant and conductivity.

Citation: Ischuk I.N., Tyapkin V.N., Postnov K.V., Panov S.A. The use of thermal imaging in solving problems of modeling radar images, J. Sib. Fed. Univ. Eng. technol., 2017, 10(2), 191-199. DOI: 10.17516/1999-494X-2017-10-2-191-199.

(C) Siberian Federal University. All rights reserved

* Corresponding author E-mail address: konstantin_postnov_88@mail.ru 


\title{
Применение тепловой томографии в решении задач моделирования радиолокационных изображений
}

\author{
И.Н. Ищука, ${ }^{a, \tilde{\sigma}}$ В.Н. Тяпкин ${ }^{\sigma}$, \\ К.В. Постнов ${ }^{\text {a }, ~ С . А . ~ П а н о в ~}{ }^{\mathrm{a}}$ \\ ${ }^{a}$ Военный учебно-научный центр Военно-воздушных сил \\ «Военно-воздушная академия \\ имени профессора Н.Е. Жуковского и Ю.А. Гагарина» \\ Россия, 394064, Воронеж, ул. Старых Большевиков, 54 а \\ ${ }^{6}$ Сибирский федеральный университет \\ Россия, 660041, Красноярск, пр. Свободньій, 79
}

В статье представлена методика получения РЛИ земной поверхности путем преобразования изображения тепловой томограммы на основании функииональной зависимости диэлектрической проницаемости материалов от их тепловых параметров. Представлена связь коэффициента поглощения электромагнитных волн и теплофизических характеристик диэлектрика.

Ключевые слова: тепловая томограмма, модель радиолокационного изображения, коэффициент отражения, диэлектрическая проницаемость, теплопроводность.

\section{Введение}

Развитие методов цифрового моделирования полей целей и сигналов оптических и радиолокационных информационных систем дистанционного зондирования характеризуется как достаточно крупными достижениями в области теоретических исследований, так и практическими результатами по созданию программно-моделирующих комплексов адекватных данным натурных измерений. На рынке компьютерных программ представлены программные продукты для расчета эффективной площади рассеивания объектов, преимущественно зарубежные: CST Microwave Studio, EMPro (разработки Agilent Technologies), HFSS. Существуют и отечественные аналоги: FEKO, «Анализ радиолокационных характеристик объектов сложной формы» (разработки МГТУ им. Н.Э. Баумана), «Банк РЛ» (разработки АО «Концерн радиостроения «ВЕГА» филиал в г. Санкт-Петербурге) [1]. Результаты методологии цифрового моделирования полей рассеяния электромагнитных волн на объектах локации естественного и антропогенного происхождения, учитывающих случайно распределенный характер рассеивающих свойств целей, представлены в ряде работ отечественных и зарубежных авторов [2-4].

Разработанные аппаратно-программные комплексы имитационного цифрового моделирования характеристик отражательных свойств целей, а также реализаций сигналов однои двухпозиционных оптических и радиолокационных систем заменяют дорогостоящие полигонные измерения универсальным и гибким вычислительным экспериментом. Однако на современном этапе развития методического аппарата моделирования радиолокационных изображений (РЛИ) остро стоит задача построения таких моделей для протяженных участков земной поверхности, способных с минимальными искажениями отображать распределения поля яркостей для текущего момента времени с высокоточными данными геометрии техногенных 
объектов и целей. Одним из способов построения таких высокоточных моделей является применение специального программного обеспечения, реализующего пространственную обработку полей и целей для заданных участков местности по изображениям, полученным в видимом диапазоне длин волн с учетом матриц высот и базовых сплошных покрытий высокого разрешения. Для повышения достоверности моделей РЛИ изображения оптического диапазона длин волн должны быть классифицированы по видам материалов. Решение данной задачи в инфракрасном диапазоне длин волн представлено в работе [5].

\section{Теоретические исследования}

При прохождении электромагнитной волны через диэлектрик часть ее энергии тратится на поляризацию материала, при этом релаксационная поляризации сопровождается электрострикцией (деформацией) диэлектрика, которая пропорциональна квадрату напряженности электрического поля. В изотропных средах электрострикция описывается законом [6]

$$
\frac{\Delta \mathrm{V}}{\mathrm{V}}=\Omega \mathrm{P}^{2}
$$

где $\Delta \mathrm{V} / \mathrm{V}$ - относительная объемная деформация; $\Omega$ - электрострикционный коэффициент, характеризующий деформацию под действием индукционного поля; Р - поляризация.

Под действием переменного электрического поля частотой $\omega$ диэлектрик в результате электрострикции колеблется с частотой $2 \omega$ за счет двукратной (за период поля) переориентации доменов, следовательно, происходит преобразование электрических колебаний в звуковые.

Известно, что коэффициент поглощения звука для поперечных волн (возбуждаемых под действием переменного электрического поля электромагнитной волны, когда время релаксации велико по сравнению с периодом колебаний в волне) пропорционален выражению [7]

$$
\mathrm{K}_{\Pi} \sim \frac{\mathrm{T}_{\mathrm{o}} \alpha^{2} \rho \mathrm{c}_{\mathrm{t}}}{\mathrm{AC}} \sqrt{\mathrm{a} \omega_{\mathrm{t}}}
$$

где $\mathrm{T}_{\mathrm{o}}$ - значение температуры твердого материала без внешнего воздействия; $\alpha-$ коэффициент термического расширения; $\rho$ - плотность; $\mathrm{c}_{\mathrm{t}}$ - скорость распространения волны; $\omega_{\mathrm{t}}-$ частота колебаний звуковой волны; А - среднее значение межатомных расстояний; $\mathrm{C}$ - теплоемкость; $\mathrm{K}_{\Pi}-$ коэффициент поглощения.

Скорость распространения электромагнитной волны зависит от показателя преломления $n$ среды и определяется выражением

$$
\mathrm{c}_{\mathrm{t}}=\mathrm{c} / \mathrm{n} \text {, }
$$

где $c$ - скорость света;

$$
\mathrm{n}=\sqrt{\frac{\varepsilon}{2}+\sqrt{\left(\frac{\varepsilon}{2}\right)^{2}+\left(\frac{2 \pi \sigma}{\omega}\right)^{2}}} ;
$$

$\sigma$ - проводимость, $\varepsilon$ - диэлектрическая проницаемость [7].

$$
-193-
$$


Для диэлектрических сред $\frac{2 \pi \sigma}{\omega}<<1$, и, следовательно, показатель преломления для диэлектрика (4) $\mathrm{n} \approx \sqrt{\varepsilon \infty}$, а выражение (3) имеет вид

$$
\mathrm{c}_{\mathrm{t}}=\mathrm{c} / \sqrt{\varepsilon \infty} \text {. }
$$

Согласно определению теплового расширения

$$
\alpha=\frac{\mathrm{V}^{\prime}-\mathrm{V}}{\mathrm{V}\left(\mathrm{T}_{2}-\mathrm{T}_{1}\right)},
$$

где $\mathrm{V}$ - объем твердого тела при температуре $\mathrm{T}_{1} ; \mathrm{V}^{\prime}$ - объем твердого тела при температуре $\mathrm{T}_{2}$, $\mathrm{T}_{2}>\mathrm{T}_{1}$. Используя (1) и введя подстановку $\Delta \mathrm{T}=\mathrm{T}_{2}-\mathrm{T}_{1}$, получим:

$$
\alpha=\frac{\mathrm{P}^{2} \Omega}{\Delta \mathrm{T}} .
$$

Тогда, произведя замену $\mathrm{C}=\lambda$ /ар, расчетное выражение для коэффициента поглощения можно привести к виду

$$
\mathrm{K}_{\Pi}=\kappa \frac{\mathrm{c} \Omega^{2} \mathrm{~T}_{\mathrm{o}} \rho \mathrm{a}^{\frac{3}{2}}}{\mathrm{~A} \Delta \mathrm{T}^{2} \lambda \varepsilon^{\frac{1}{2}}} \mathrm{P}^{4} \sqrt{2 \omega},
$$

где $\kappa$ - параметр пропорциональности [7].

Следовательно, выражение (8) устанавливает связь между $\mathrm{K}_{\text {п }}$ и теплофизическими параметрами (теплопроводность и температуропроводность) диэлектрических материалов. С использованием данной зависимости становится возможным по данным дистанционного измерения распределения теплофизических параметров приповерхностного слоя Земли и расположенных на ней техногенных объектов построить приближенную модель РЛИ.

Теоретические и экспериментальные результаты определения пространственного распределения теплофизических параметров ( $\lambda$ и а), полученные с использованием БЛА на основе решения задачи редукции кубоида ИК-изображений, представлены в работах $[8,9]$

\section{Методика обработки изображений}

Методика обработки кубоида ИК-изображений исследуемой поверхности заключается в выполнении следующих действий:

1. Получение тепловых томограмм поверхности $[10,11], \mathrm{K}_{\text {п }}$ которого является «эталонным». Полученные тепловые томограммы будут «эталонными» $\mathrm{T}_{\text {аэ }}, \mathrm{T}_{\lambda_{\ni}}$.

2. Получение тепловых томограмм исследуемой поверхности и при тех же режимных параметрах проведение измерений, что и в пункте $1-\mathrm{T}_{\mathrm{a}}, \mathrm{T}_{\lambda}$.

3. Преобразование тепловых томограмм по температуропроводности и теплопроводности в изображение, имеющее размерность коэффициента поглощения электромагнитной волны (ЭМВ). Используя принцип эталонирования, на основании полученных тепло-

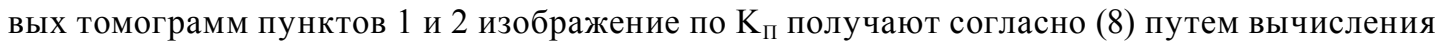
отношения 


$$
\mathrm{K}_{\Pi}[\mathrm{i}, \mathrm{j}]=\frac{\lambda[\mathrm{i}, \mathrm{j}] \mathrm{a}_{\ni}^{\frac{3}{2}}[\mathrm{i}, \mathrm{j}]}{\lambda_{\ni}[\mathrm{i}, \mathrm{j}] \mathrm{a}^{\frac{3}{2}}[\mathrm{i}, \mathrm{j}]},
$$

где $\mathrm{i}, \mathrm{j}$ - номера отсчетов пикселей на изображении.

В свою очередь, диэлектрические немагнитные материалы поглощают не весь объем электромагнитной энергии, часть ее отражается. Характер отражения радиоволн от земной поверхности зависит в первую очередь от электрических свойств и степени неровности (шероховатости) этой поверхности, а также от длины волны, поляризации векторов электромагнитного поля и угла падения облучающих электромагнитных колебаний.

Коэффициент отражения электромагнитных волн приповерхностного слоя Земли и поверхностных объектов определяется диэлектрической проницаемостью. Для случая нормального падения плоской волны на немагнитный диэлектрик без потерь он будет описываться выражением

$$
\mathrm{K}_{\mathrm{OT}}=\frac{(1-\sqrt{\varepsilon})^{2}}{(1+\sqrt{\varepsilon})^{2}} .
$$

Когда плоская электромагнитная волна, распространяясь в среде, падает на границу раздела с диэлектрической средой под некоторым углом падения $\varphi,(\varphi \square[0, \pi / 2])$, коэффициент отражения будет зависеть от ориентации плоскости поляризации ЭМВ и плоскости падения ЭМВ. При перпендикулярной поляризации, когда плоскость поляризации, содержащая направление вектора напряженности электрического поля Е, перпендикулярна плоскости падения волны, коэффициент отражения для немагнитного диэлектрика будет определяться выражением

$$
\mathrm{K}_{\mathrm{OT}}=\frac{\left(\cos \varphi-\sqrt{\varepsilon-\sin ^{2} \varphi}\right)^{2}}{\left(\cos \varphi+\sqrt{\varepsilon-\sin ^{2} \varphi}\right)^{2}}
$$

а при параллельной поляризации [12] -

$$
\mathrm{K}_{\mathrm{OT}}=\frac{\left(\sqrt{\varepsilon-\sin ^{2} \varphi}-\cos \varphi\right)^{2}}{\left(\sqrt{\varepsilon-\sin ^{2} \varphi}+\cos \varphi\right)^{2}} .
$$

Значения теплопроводности $\lambda$ и диэлектрической проницаемости $\varepsilon$ некоторых материалов для длины волны, равной 3 см, представлены в табл. 1 .

На основании табличных данных зависимость диэлектрической проницаемости от теплопроводности для соответствующих материалов можно аппроксимировать сплайнинтерполированным полиномом $\varepsilon=\mathrm{f}_{\varepsilon}(\lambda)$ (рис. 1$)$.

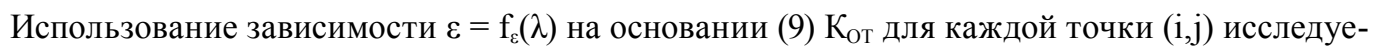
мой поверхности будет описываться выражениями

$$
\begin{gathered}
\mathrm{K}_{\mathrm{OT}}[\mathrm{i}, \mathrm{j}]=\frac{\left(\cos \varphi-\sqrt{\left.\mathrm{f}_{\varepsilon}(\lambda[\mathrm{i}, \mathrm{j}])-\sin ^{2} \varphi\right)^{2}}\right.}{\left(\cos \varphi+\sqrt{\mathrm{f}_{\varepsilon}(\lambda[\mathrm{i}, \mathrm{j}])-\sin ^{2} \varphi}\right)^{2}} \\
-195-
\end{gathered}
$$




$$
K_{\mathrm{OT}}[\mathrm{i}, \mathrm{j}]=\frac{\left(\sqrt{\mathrm{f}_{\varepsilon}(\lambda[\mathrm{i}, \mathrm{j}])-\sin ^{2} \varphi}-\cos \varphi\right)^{2}}{\left(\sqrt{\mathrm{f}_{\varepsilon}(\lambda[\mathrm{i}, \mathrm{j}])-\sin ^{2} \varphi}+\cos \varphi\right)^{2}}
$$

для соответствующих направлений вектора поляризации $\mathrm{E}$.

Следовательно, используя изображения тепловых томограмм, полученных в результате дистанционного мониторинга земной поверхности с БЛА в ИК-диапазоне длин волн, на основании (13) и (14) строят приближенную модель РЛИ.

\section{Результаты численного эксперимента}

Для численной апробации представленной методики построения РЛИ в среде теплового моделирования RadTherm была разработана пространственная тепловая модель местности с известными теплофизическими характеристиками. Внешний вид тепловой модели изображен на рис. 2.

Тепловая модель включает поле, примыкающую к нему реку, дорогу, проходящую через поле, и мост через реку, а также три объекта военной техники. В результате обработки пространственной тепловой модели было получено изображение поля яркости тепловой томограммы, кластеризированное по теплопроводности [4] (рис. 3).

Таблица 1 Значение теплопроводности и диэлектрической проницаемости для некоторых материалов при длине ЭМВ 3 см

\begin{tabular}{|c|l|c|c|}
\hline Номер & \multicolumn{1}{|c|}{ Материал } & $\lambda$ & $\boldsymbol{\varepsilon}$ \\
\hline 1 & Керамзит & 0,18 & 2,1 \\
\hline 2 & Гравий & 0,2 & 2,6 \\
\hline 3 & Древесина (береза) & 0,25 & 2 \\
\hline 4 & Полиэтилен & 0,4 & 2,7 \\
\hline 5 & Кирпич красный & 0,56 & 3,7 \\
\hline 6 & Известняк & 0,8 & 4,3 \\
\hline 7 & Асфальтобетон & 1,05 & 4,3 \\
\hline 8 & Мрамор & 2,91 & 2,4 \\
\hline 9 & Теплопроводник & 15 & 0,25 \\
\hline
\end{tabular}

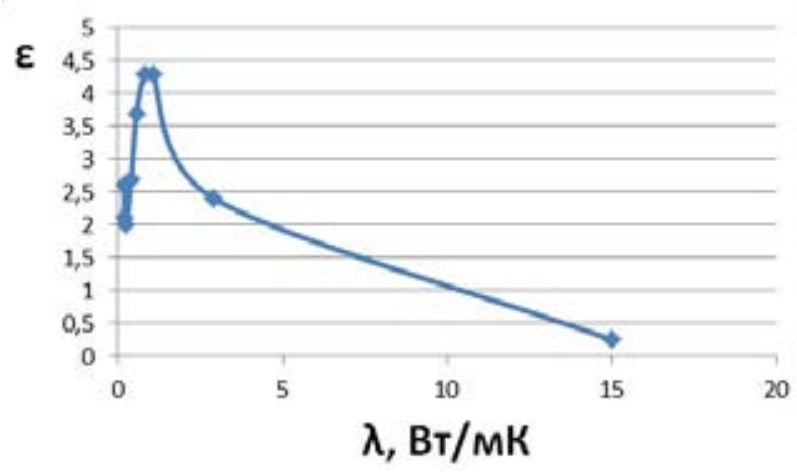

Рис. 1. График аппроксимированной функциональной зависимости 


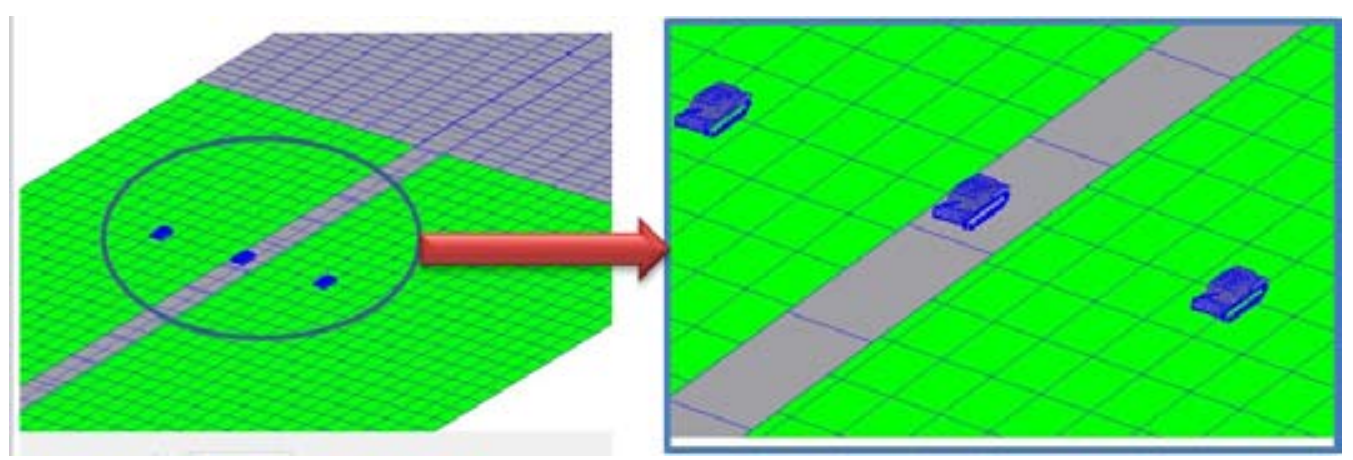

Рис. 2. Пространственная тепловая модель местности

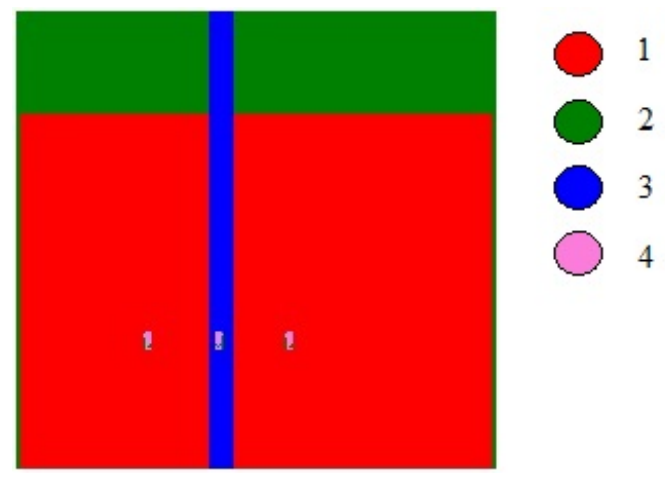

Рис. 3. Изображение поля яркости тепловой томограммы, кластеризированное по теплопроводности: $1-$ земля $(\lambda=1 \mathrm{BT} / \mathrm{MK}) ; 2$ - вода $(\lambda=0,559 \mathrm{BT} / \mathrm{MK}) ; 3$ - асфальтовая дорога $(\lambda=0,72 \mathrm{BT} / \mathrm{MK}) ; 4$ - металлический объект военной техники $(\lambda=15 \mathrm{BT} / \mathrm{MK})$

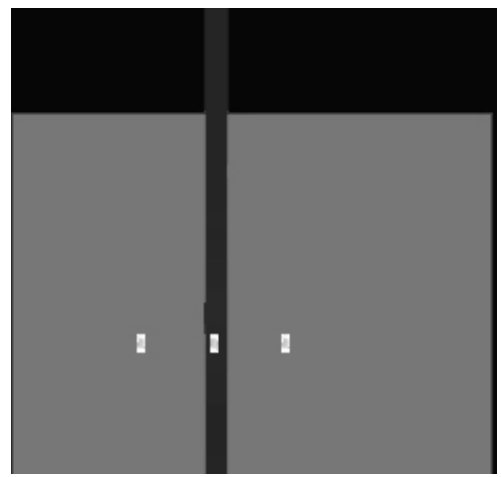

Рис. 4. Модель РЛИ

На рис. 3 классифицированы земля, вода, асфальтовая дорога и три объекта военной техники.

На основании зависимости (13) пространственное распределение теплопроводности, представленное в виде поля яркости тепловой томограммы (рис. 3), получена приближенная модель РЛИ (рис. 4), адекватно отражающая пространственное распределение Кот. 


\section{Вывод}

Таким образом, в статье представлена методика получения РЛИ земной поверхности путем преобразования изображения тепловой томограммы на основании функциональной зависимости $\varepsilon=\mathrm{f}_{\varepsilon}(\lambda)$ при длине ЭМВ, равной 3 см. Данная методика обеспечивает получение модели РЛИ, приближенной к реальным условиям наблюдения, полученной в результате дистанционного мониторинга земной поверхности и техногенных объектов с помощью тепловизионного приемника, размещенного на БЛА в пассивном режиме.

\section{Работа выполнена при финансовой поддержке РФФИ (грант № 15-08-02611 А).}

\section{Список литературы}

[1] Лобанов Б. С., Дамарацкий И. А., Миронов Ю. М. Оценка точности современных компьютерных программ для вычисления эффективной поверхности рассеяния объектов. Наука и образование: научное издание МГТУ им. Н.Э. Баумана, 2013, 9, 455-478 [Lobanov B.S., Damaratsky I.A., Mironov Y.M. Assessment of the accuracy of modern computer programs to calculate the effective surface scattering objects. Science and education: scientific publication MSTU. NE Bauman, 2013, 9, 455-478 (in Russian)].

[2] Коренной А.В., Лепёшкин С.А., Кадочников А.П., Ященко Е.А. Моделирование радиолокационных изображений на основе стохастических дифференциальных уравнений в частных производных. Радиотехника, 2016, 10, 134-143 [Korennoy A.V., Lepyoshkin S.A., Kadochnikov A.P., Yaschenko E.A., Simulation of radar images based on stochastic differential equations partial. Radio Engineering, 2016, 10, 134-143 (in Russian)].

[3] Лобунец Л. В., Решетко А. Д. Цифровое моделирование радиоизображений объектов локации с шероховатой поверхностью. Научный вестник МГТУ ГА. Радиофизика и радиотехника, 2001, 39, 45-54. [Lobunets L.V., Reshetko A.D., Numerical simulation of radio images of objects ranging from a rough surface. Scientific Bulletin MSTUCA. Radio Physics and Radio Engineering, 2001, 39, 45-54. (in Russian)].

[4] Demirci Sevket, Ozdemir Caner, Akdagli Ali, Yigit Enes Clutter reduction in synthetic aperture radar images with statistical modeling: An application to MSTAR data. Microwave \& Optical Technology Letters, 2008, 50 (6), 1514-1520.

[5] Ищук И.Н., Филимонов А.М., Степанов Е.А., Постнов К.В. Способ классификации стационарных и квазистационарных объектов по данным динамических инфракрасных изображений, получаемых комплексами с беспилотными летательными аппаратами. Радиотехника, 2016, 10, 145-153 [Ischuk I.N., Filimonov A.M., Stepanov E.A., Postnov K.V. The method of classification of stationary and quasi-stationary objects according to the dynamic infrared images obtained complexes with unmanned aerial vehicles. Radio Engineering, 2016, 10, 145-153 (in Russian)].

[6] Ландау Л.Д., Лифшиц Е.Н. Электродинамика сплошных сред. М.: Гостехиздат, 1957. 532 c. [Landau L.D., Lifshitz E.N. Electrodynamics of Continuous Media. M .: Gostekhizdat, 1957. 532 p. (in Russian)].

[7] Фесенко А. И., Ищук И. Н., Штейнбрехер В. В. Методы и приборы технического диагностирования тепловой защиты и радиопоглощающих покрытий авиационно-космических аппаpaтов. М.: Машиностроение, 2008. 200 с. [Fesenko A.I., Ischuk I.N., Steinbrecher V.V., Methods 
and devices of technical diagnostics and thermal protection of radar absorbing coatings aerospace vehicles. Moscow, Engineering, 2008. 200 p. (in Russian)].

[8] Ishchuk I.N., Filimonov A.M., Tyapkin V.N., Semenov M.E., Kabulova E.A. Cuboids of Infrared Images Reduction Obtained from Unmanned Aerial Vehicles. Modern applied science, 2015, 3(9), 233-240.

[9] Ishchuk, I.N., Parfiriev, A.V. The Reconstruction of a Cuboid of Infrared Images to Detect Hidden Objects. Part 1. A Solution Based on the Coefficient Inverse Problem of Heat Conduction. Measurement Techniques, 2014, 56 (10), 1162-1166.

[10] Ishchuk, I.N., Parfiriev, A.V. The Reconstruction of a Cuboid of Infrared Images to Detect Hidden Objects. Part 2. A Method and Apparatus for Remote Measurements of the Thermal Parameters of Isotropic. Materials. Measurement Techniques, 2014, 57(1), 74-78.

[11] Ищук И.Н., Обухов В.В., Парфирьев А.В., Филимонов А.М. Методика дистанционного контроля изотропных материалов путем редукции кубоида ИК-изображений. Измерительная техника, 2014, 9, 41-45. [Ischuk I.N., Obukhov V.V., Parfiriev A.V. Filimonov A.M., Methods of remote monitoring isotropic materials by reduction cuboid IR images. Measuring equipment, in 2014, 9, 41-45. (in Russian)].

[12] Пенской А.С., Мальцев Н.И., Пустовалов А.П. Измерения коэффициента отражения воды в СВЧ-диапазоне. Известия Волгоградского государственного технического университе$m a, 2013,7,3(106), 91-95$ [Penskoi A.S., Maltsev N.I., Pustovalov A.P. Measurements of the reflection coefficient of water in the microwave range. News of Volgograd State Technical University, 2013, 7, 3 (106), 91-95 (in Russian)]. 